\title{
PTEN in propofol-induced insulin resistance in mouse primary hepatocytes
}

\author{
LONG ZHOU ${ }^{1,2^{*}}$, LILIN WANG ${ }^{3^{*}}$, XUHUAI HU ${ }^{2}$ and YUANTAO LI ${ }^{1}$ \\ ${ }^{1}$ Department of Anesthesiology, Shenzhen Maternity and Child Healthcare Hospital, Southern Medical University; \\ ${ }^{2}$ Shenzhen Health Development Research Center; ${ }^{3}$ Shenzhen Blood Center, Shenzhen, Guangdong 518000, P.R. China
}

Received May 13, 2018; Accepted August 31, 2018

DOI: $10.3892 /$ etm.2018.6815

\begin{abstract}
Propofol is the most common intravenous anesthetic agent used in clinical practice. Propofol can induce insulin resistance in mouse primary hepatocytes, however the molecular mechanism through which propofol acts remains largely unknown. Based on previous studies, it was hypothesized that phosphatase and tensin homolog (PTEN) is involved in propofol-mediated insulin resistance. The aim of the present study was to investigate the biological function of PTEN and its molecular mechanism in propofol-induced insulin resistance in mouse primary hepatocytes. Mouse primary hepatocytes were treated with propofol and transfected with small interfering RNA (siRNA)-996 to silence the endogenous expression of PTEN. The current study assessed the effects of propofol and PTEN knockdown on the expression of PTEN and several key enzymes of the phosphoinositide 3-kinase/protein kinase B/glycogen synthase kinase- $3 \beta$ signaling pathway, as well as the glycogen content in mouse primary hepatocytes. Treatment with propofol significantly increased protein and mRNA PTEN expression in mouse primary hepatocytes. In addition, knockdown of PTEN reversed propofol-induced insulin resistance in mouse primary hepatocytes. The present study indicated that PTEN serves a role in the physiological process of propofol-induced insulin resistance in mouse primary hepatocytes, and PTEN inhibition may be a potential target for therapeutic intervention against propofol-induced adverse effects.
\end{abstract}

\section{Introduction}

Propofol is widely used for general anesthesia or sedation in critically ill patients (1). Intensive insulin therapy can reduce

Correspondence to: Dr Lilin Wang, Shenzhen Blood Center, 2 Meigang South Street, Shenzhen, Guangdong 518000, P.R. China

E-mail: 1ilywang0724@163.com

*Contributed equally

Key words: phosphatase and tensin homolog, propofol, mouse primary hepatocytes, glycogen, protein kinase B, glycogen synthase kinase-3 $\beta$ morbidity and mortality in patients in surgical intensive care units, and, therefore, insulin resistance is an important factor affecting the prognosis of critically ill patients $(2,3)$. It has been reported that anesthesia with propofol could induce systemic insulin resistance and decrease insulin-stimulated glucose uptake in skeletal and heart muscles and attenuate the insulin-mediated suppression of hepatic glucose output in rats, however the specific molecular mechanisms underlying this phenomenon remain unknown (4). Previous studies have revealed that propofol can inhibit the phosphoinositide 3-kinase (PI3K)/protein kinase $\mathrm{B}$ (Akt)/glycogen synthase kinase (GSK)-3b signaling pathway and glycogen synthesis in mouse primary hepatocytes, and the target of propofol-induced insulin resistance in primary mouse hepatocytes was suggested to be upstream of GSK-3 $\beta$ (5). In this study cell viability was assessed by MTT reduction assay, as previously described (5). Propofol was added at a final concentration of $10 \mu \mathrm{g} / \mathrm{ml}$, based on the results of previous studies (5-8). PTEN is an important regulatory gene of the PI3K/Akt/GSK-3 $\beta$ signaling pathway (9-11), and inhibition of PTEN activity can activate the Akt signal transduction pathway (12). RNA interference is the process of sequence-specific, post-transcriptional gene silencing in animals and plants, initiated by double-stranded RNA (dsRNA) homologous in sequence to the silenced gene (13). The mediators of sequence-specific messenger RNA degradation are 21- and 22-nucleotide long small interfering RNAs (siRNAs) generated by ribonuclease III cleavage from longer dsRNAs (13). The current study used siRNA-996 to silence the endogenous PTEN gene expression, observed the alterations of the signaling pathway and glycogen synthesis in primary mouse hepatocytes, and investigated the role of PTEN in propofol-induced insulin resistance. The present study demonstrated that propofol enhanced PTEN expression in mouse primary hepatocytes. In addition, PTEN knockdown reversed propofol-induced inhibition of the PI3K/Akt/GSK-3 $\beta$ signaling pathway and glycogen synthesis in mouse primary hepatocytes. These results indicated that PTEN may be the target of propofol-induced insulin resistance in mouse primary hepatocytes, and, therefore, PTEN could be a potential target for therapeutic intervention against propofol-induced adverse effects.

\section{Materials and methods}

Animals. Male C57BL/6J mice ( $\mathrm{n}=10$; age, 8 weeks; weight, 24-28 g) were provided by Peking University Health Science 
Center (Beijing, China). Mice were housed at a constant temperature $\left(22 \pm 2^{\circ} \mathrm{C}\right)$ and $55 \pm 10 \%$ relative humidity with a $12 \mathrm{~h}$ light/dark cycle and free access to food and water. The mice were fasted for $12 \mathrm{~h}$ prior to all experiments. Animal procedures were performed in accordance with the National Institutes of Health Animal Care and Use Guidelines (14), and animal experimental protocols were approved by the Ethics Committee of Shenzhen Maternity and Child Healthcare Hospital (Shenzhen, China).

Isolation of mouse primary hepatocytes. Primary hepatocytes were isolated using a two-step collagenase perfusion method, as previously described $(15,16)$. The hepatocytes were plated in collagen-coated $25-\mathrm{cm}^{2}$ flasks at a density of $1 \times 10^{6}$ cells/flask and were used as the control group in the following experiment. Dimethylsulfoxide (DMSO) at a final concentration of $0.1 \%$ was added to the DMSO group cells.

Western blot analysis. Protein was extracted with radioimmunoprecipitation assay lysis buffer (Thermo Fisher Scientific, Inc., Waltham, MA, USA). The protein content of cells was assessed using Pierce BCA protein assay kit (Thermo Fisher Scientific Inc.). The proteins (15-30 $\mu \mathrm{g} /$ lane) were separated on SDS-PAGE $10 \%$ gels (Bio-Rad Laboratories, Inc., Hercules, CA, USA) and transferred onto a polyvinylidene difluoride membranes (EMD Millipore; Billerica, MA, USA). The membranes were blocked with $5 \%$ nonfat dry milk at $4^{\circ} \mathrm{C}$ overnight, and incubated with primary antibodies against PTEN (cat. no. 9188), Akt (cat. no. 9272), phosphorylated Akt (cat. no. 9271), GSK (cat. no. 9315), phosphorylated GSK (cat. no. 9323) and $\beta$-actin (cat. no. 4970; all 1:1,000; Cell Signaling Technology, Inc., Danvers, MA, USA) overnight at $4^{\circ} \mathrm{C}$. Following primary incubation, membranes were incubated with horseradish peroxidase-conjugated goat anti-rabbit secondary antibody (1:5,000; cat. no. ab6721; Abcam) at room temperature for $2 \mathrm{~h}$. The blots were visualized using an enhanced chemiluminescence detection system (EMD Millipore) and quantified by densitometry using Image-Pro Plus software 6.0 (Media Cybernetics, Inc., Rockville, MD, USA). $\beta$-actin was used as the internal control.

Reverse transcription-quantitative polymerase chain reaction (RT-qPCR). Total RNA was extracted using TRIzol ${ }^{\circledR}$ reagent (Invitrogen; Thermo Fisher Scientific, Inc., Waltham, MA, USA), according to the manufacturer's protocol. Total RNA was reverse transcribed into cDNA using the M-MLV reverse transcriptase kit (Promega Corporation, Madison, WI, USA), according to the manufacturer's protocol. qPCR was subsequently performed using the $\mathrm{SYBR}^{\circledR}$-Green PCR mastermix (Takara Biotechnology Co., Ltd., Dalian, China), according to manufacturer's protocol The following primer pairs were used for the qPCR: hypoxanthine phosphoribosyltransferase 1 (HPRT1) forward, 5'-AATTATGGACAGGACTGAACGTCTTGCT-3' and reverse, 5'-TCCAGCAGGTCAGCAAAGAATTTATAG C-3'; and mouse PTEN forward, 5'-AATTCCCAGTCAGAG GCGCTATGT-3' and reverse, 5'-GATTGCAAGTTCCGCCAC TGAACA-3'. The following thermocycling conditions were used for the qPCR: Initial denaturation at $95^{\circ} \mathrm{C}$ for $10 \mathrm{~min}$; 40 cycles of $95^{\circ} \mathrm{C}$ for $15 \mathrm{sec}$ and $60^{\circ} \mathrm{C}$ for $1 \mathrm{~min}$. The relative mRNA levels were quantified using the $2^{-\Delta \Delta \mathrm{Cq}}$ method (17) and normalized to the reference gene HPRT1-R.
Cell culture and transfections. Mouse primary hepatocytes were plated on 24-well plates at a density of $6 \times 10^{4}$ cells/well and grown in Dulbecco's modified Eagle's medium (DMEM; Invitrogen; Thermo Fisher Scientific, Inc.) supplemented with $10 \%$ heat-inactivated fetal bovine serum (FBS; Thermo Fisher Scientific, Inc.) and $100 \mathrm{U} / \mathrm{ml}$ penicillin, at $37^{\circ} \mathrm{C}$ in a $5 \% \mathrm{CO}_{2}$-humidified incubator. The siRNA (GenePharma Co., Ltd., Shanghai, China) sequences used in this study are as follows. si-PTEN (Cy3-labeled siR-996) sense, 5'-GGUGUA UACAGGAACAAUATT-3' and anti-sense, 5'-UAUUGUUCC UGUAUACACCTT-3'; negative control siRNA sense, 5'-UUC UCCGAACGUGUCACGUTT-3' and antisense, 5'-ACGUGA CACGUUCGGAGAATT-3'. A total of $100 \mu 1$ siR-996 at a final concentration of $50 \mathrm{nmol} / \mathrm{l}$ was mixed with $3 \mu \mathrm{l}$ HiPerFect Transfection reagent (Qiagen China Co., Ltd., Shanghai, China) in serum-free DMEM at room temperature for $10 \mathrm{~min}$, then the cells were transfected for $24 \mathrm{~h}$ at $37^{\circ} \mathrm{C}$. Negative control siRNA was added at a final concentration of $50 \mathrm{nmol} / \mathrm{l}$ in the negative control (NC) group. Cells were incubated with $2 \mu \mathrm{g} / \mathrm{ml}$ Hoechst 33342 solution (Sigma-Aldrich; Merck KGaA, Darmstadt, Germany) for $10 \mathrm{~min}$ at room temperature and washed twice with PBS. The transfection efficiency determined by counting the red fluorescent Cy3-labeled siR-996 in transfected cells under an inverted fluorescent microscope (Nikon Corporation, Tokyo, Japan).

Analysis of glycogen content. Glycogen levels were measured following incubation of cells with $10 \mathrm{nmol} / \mathrm{l}$ insulin (United States Biological, Salem, MA, USA) at room temperature for 3 h using a glycogen assay kit (BioVision, Inc., Milpitas, CA, USA).

Statistical analysis. The results are reported as the mean \pm standard deviation of at least three independent experiments with a minimum sample size of three. Statistical analysis was performed with SPSS software (version 19.0; IBM Corp., Armonk, NY, USA). All experimental data were analyzed using one-way analysis of variance followed by Tukey's test to confirm statistical differences among multiple groups. $\mathrm{P}<0.05$ was considered to indicate a statistically significant difference.

\section{Results}

Propofol enhances PTEN expression in mouse primary hepatocytes. The effect of treatment with propofol on PTEN protein and mRNA expression in mouse primary hepatocytes was analyzed using western blotting and RT-qPCR, respectively. Treatment with propofol significantly increased PTEN protein and mRNA expression compared with the control group (Fig. 1).

Transfection efficiency of Cy3-siRNA. Mouse primary hepatocytes were transfected with Cy3-labelled siR-996. Cells were initially observed under an inverted fluorescent microscope. The subcellular localization and distribution of cells transfected with Cy3-labelled siRNA were observed using the fluorescent microscope. Transfection efficiency of Cy3-labelled siRNA was $>95 \%$ in mouse primary hepatocytes, $24 \mathrm{~h}$ after transfection (Fig. 2). 

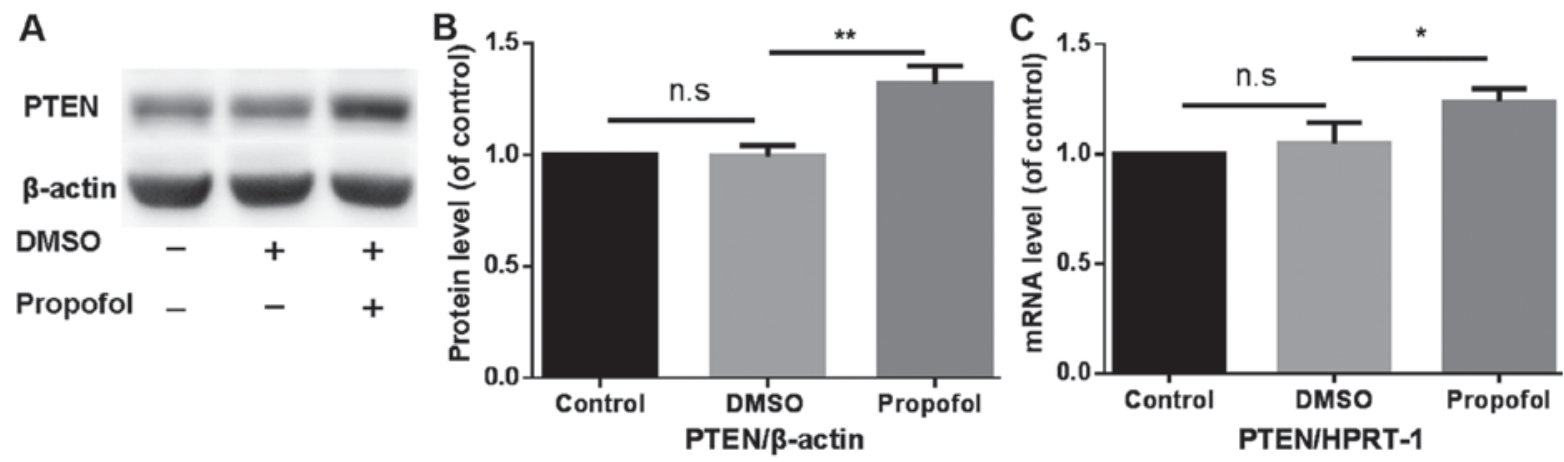

Figure 1. Propofol enhances PTEN expression in mouse primary hepatocytes. (A) The protein expression level of PTEN was determined by western blot analysis. $\beta$-actin was used as the loading control. (B) Propofol significantly increased PTEN protein expression. Data are presented as the mean \pm standard deviation. ${ }^{* *} \mathrm{P}<0.01$ vs. DMSO group. (C) The mRNA expression level of PTEN was determined by reverse transcription-quantitative polymerase chain reaction. The mRNA expression levels were normalized to that of HPRT-1. Data are presented as the mean \pm standard deviation. "P<0.05 vs. DMSO group. PTEN, phosphatase and tensin homolog; HPRT-1, hypoxanthine phosphoribosyltransferase 1; DMSO, dimethylsulfoxide; n.s., not significant.
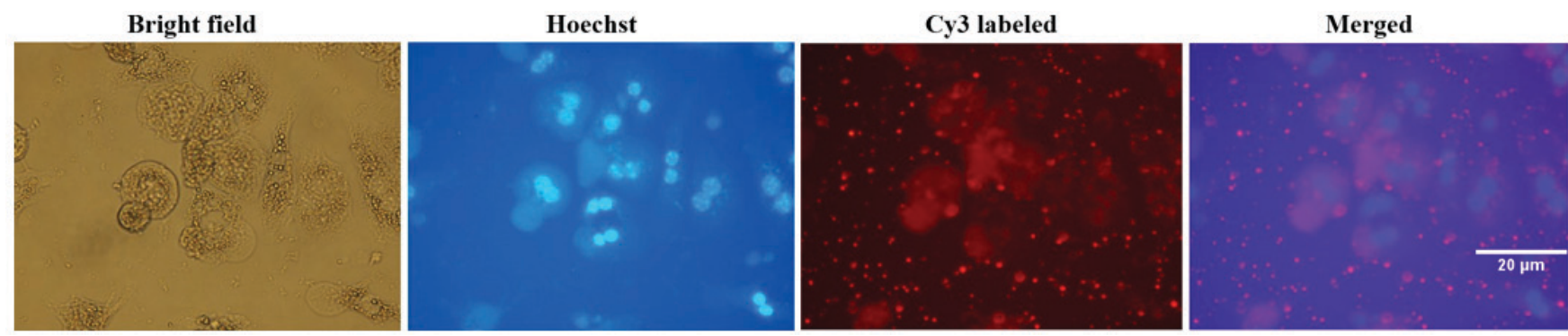

Figure 2. Subcellular localization of Cy3-siRNA in mouse primary hepatocytes (magnification, $\mathrm{x} 1,000$ ).

PTEN knockdown reverses propofol-induced inhibition of the PIJK/Akt/GSK-3 $\beta$ signaling pathway and glycogen synthesis in mouse primary hepatocytes. To further confirm the biological functions of PTEN in propofol-induced insulin resistance in mouse hepatocytes, endogenous PTEN expression was silenced by transfecting siR-996 into mouse primary hepatocytes, with simultaneous treatment with propofol (final concentration, $10 \mu \mathrm{g} / \mathrm{ml}$ ) for $24 \mathrm{~h}$. Following PTEN knockdown and treatment with propofol, western blot analysis was used to detect the protein expression levels of PTEN and components of the PI3K/Akt/GSK-3 $\beta$ signaling pathway (Fig. 3A). Compared with the NC+DMSO group, the protein expression level of PTEN in the $\mathrm{NC}+$ propofol group significantly increased $(\mathrm{P}<0.01)$, while the protein expression level of PTEN in the DMSO+siR-996 group significantly decreased $(\mathrm{P}<0.001)$ (Fig. 3B). There was no significant difference in the protein expression level of PTEN between the siR-996+Propofol group and the control group (Fig. 3B). Compared with the NC+DMSO group, the phosphorylation levels of Akt (Ser473) in the $\mathrm{NC}+$ propofol group decreased $(\mathrm{P}<0.05)$, while the phosphorylation levels of Akt (Ser473) in the DMSO+siR-996 group increased $(\mathrm{P}<0.05)$ (Fig. 3C). Compared with the $\mathrm{NC}+\mathrm{DMSO}$ group, the phosphorylation levels of GSK-3 $\beta$ (Ser9) in the NC+propofol group significantly decreased $(\mathrm{P}<0.01)$, while the phosphorylation levels of GSK-3 $\beta$ (Ser9) in the DMSO+siR-996 group increased $(\mathrm{P}<0.05)$ (Fig. 3D). There was no significant difference in the phosphorylation levels of Akt (Ser473) and GSK-3 $\beta$ (Ser9) between the siR-996+Propofol group and the control group (Fig. 3C and D). In addition, the glycogen assay kit was used to detect the level of glycogen synthesis. Compared with the NC+DMSO group, the glycogen level in the $\mathrm{NC}+$ propofol group decreased $(\mathrm{P}<0.05)$, while the glycogen level in the DMSO+siR-996 group was increased $(\mathrm{P}<0.05)$ (Fig. 3D). However, there was no significant difference in the rate of glycogen synthesis between the siR-996+Propofol group and the control group (Fig. 3E).

\section{Discussion}

Insulin resistance is a physiological condition in which normal or elevated insulin levels produce an attenuated biological effect (18). In 1988, Reaven (19) first suggested the idea of insulin resistance being a common phenomenon, which can occur in a number of pathological and physiological conditions, other than diabetes. Insulin resistance severely affects the prognosis of critically ill patients $(2,3,20)$. A clinical study revealed that intensive insulin therapy could reduce mortality by $42.5 \%$ as well as significantly reducing other complications in surgical intensive care unit patients (21). Propofol is the most common intravenous anesthetic agent used in clinical practice (1). Propofol has been demonstrated to cause systemic insulin resistance in rats (4). In addition, previous studies have indicated that propofol can induce insulin resistance in mouse primary hepatocytes (5). The molecular mechanism through which propofol influences insulin resistance in mouse primary hepatocytes remains unknown, however the present study focused on PTEN as a potential target for therapeutic intervention to treat the adverse reaction of propofol.

PTEN was first identified, cloned and named in 1997 by three independent research groups (22-24). The protein 
A

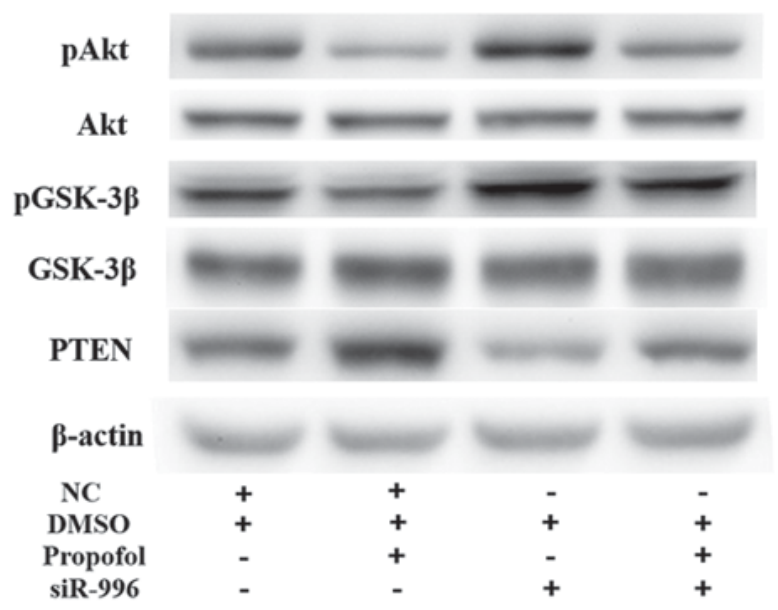

C

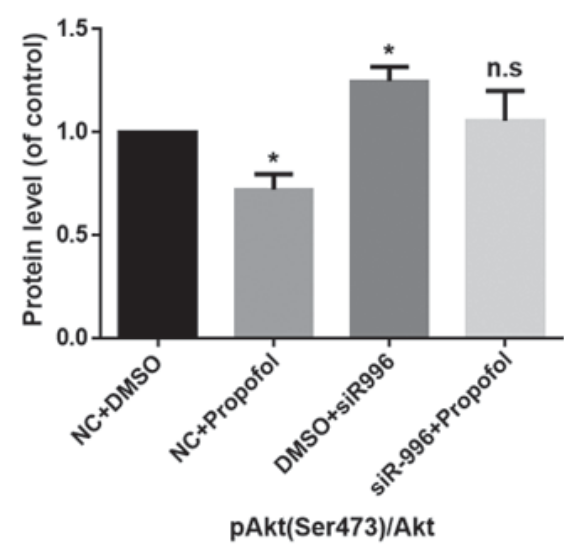

D

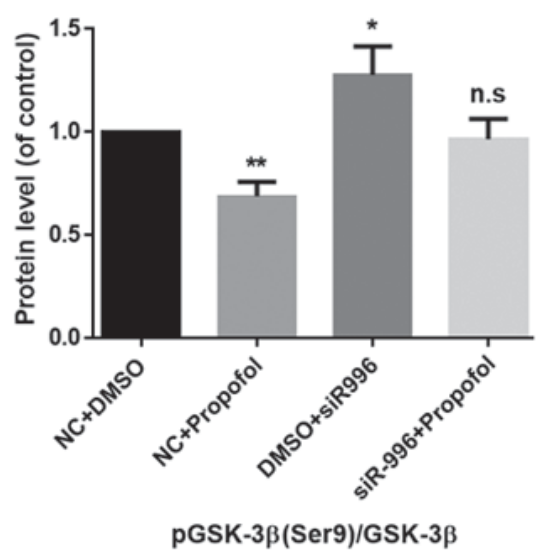

B

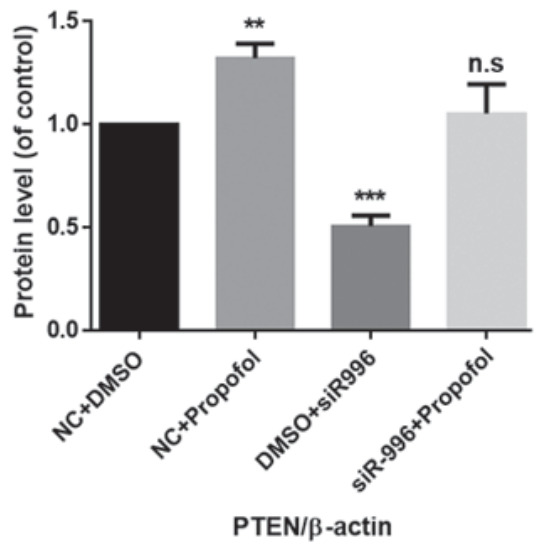

E

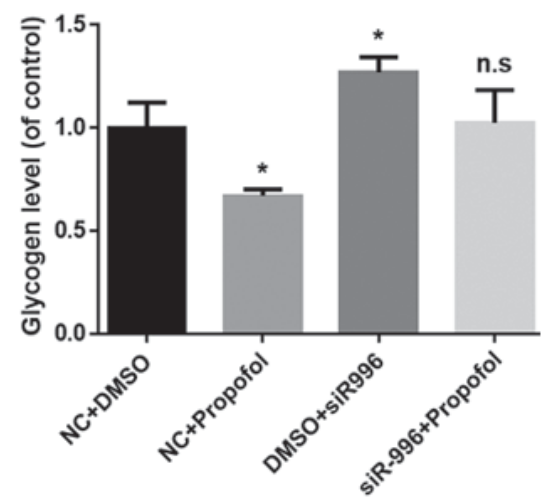

Figure 3. PTEN knockdown reverses propofol-induced insulin resistance in mouse primary hepatocytes. (A) The protein expression levels of pAkt (Ser473), Akt, pGSK-3 $\beta$ (Ser9), GSK-3 $\beta$ and PTEN were determined by western blot analysis. $\beta$-actin was used as the loading control. (B) PTEN knockdown reversed the propofol-induced enhancement of PTEN protein expression. Data are presented as the mean \pm standard deviation. ${ }^{* *} \mathrm{P}<0.01$ and ${ }^{* * *} \mathrm{P}<0.001$ vs. NC+DMSO group. (C) PTEN knockdown reversed the propofol-induced inhibition of pAkt (Ser473)/Akt. Data are presented as the mean \pm standard deviation. ${ }^{*} \mathrm{P}<0.05$ vs. NC+DMSO group. (D) PTEN knockdown reversed the propofol-induced inhibition of pGSK-3 $\beta$ (Ser9)/GSK-3 $\beta$. Data are presented as the mean \pm standard deviation. ${ }^{*} \mathrm{P}<0.05$ and ${ }^{* *} \mathrm{P}<0.01$ vs. NC+DMSO group. (E) Glycogen levels were measured in mouse primary hepatocytes using a glycogen assay kit. PTEN knockdown reversed the propofol-induced inhibition of glycogen synthesis. Data are presented as the mean \pm standard deviation. ${ }^{*} \mathrm{P}<0.05$ vs. NC+DMSO group. PTEN, phosphatase and tensin homolog; Akt, protein kinase B; GSK, glycogen synthase kinase; DMSO, dimethylsulfoxide; NC, negative control; n.s., not significant; p, phosphorylated.

encoded by PTEN is a dual-specificity phosphatase, with both lipid and protein phosphatase activity. The phosphorylation of proteins can affect a number of signal transduction pathways and regulate gene transcription in the nucleus. PTEN is the first tumor-suppressor gene identified with phosphatase activity (23). It serves a role in cell apoptosis, cell cycle arrest and cell migration (25). PTEN is an important regulator of the PI3K/Akt signaling pathway $(9-11,26)$. PTEN negatively regulates PI3K/Akt signal transduction by catalyzing the dephosphorylation of the lipid signaling intermediate phosphatidylinositol-3,4,5-trisphosphate (27). Inhibition of PTEN activity can activate the Akt signal transduction pathway (12). The present study indicated that protein and mRNA expression levels of PTEN increased in mouse primary hepatocytes following treatment with propofol for $24 \mathrm{~h}$. In addition, PTEN knockdown reversed propofol-induced inhibition of the PI3K/Akt/GSK-3 $\beta$ signaling pathway and glycogen synthesis in mouse primary hepatocytes. In conclusion, the present study suggested that PTEN may be the target of propofol-induced insulin resistance in mouse primary hepatocytes, and, therefore, could be a potential target for therapeutic prevention of propofol-induced adverse effects.

\section{Acknowledgements}

Not applicable.

\section{Funding}

The present study was supported by grants from the Shenzhen Science and Technology Plan Project (grant no. JCYJ2016 0427145626702) and Shenzhen Health Commission Project (grant no. 201605019).

\section{Availability of data and materials}

The datasets used and/or analyzed during the current study are available from the corresponding author on reasonable request. 


\section{Authors' contributions}

LZ and LW designed and performed the experiments, and wrote the manuscript. $\mathrm{XH}$ has participated in data analysis. YL has participated in the design of this experiment.

\section{Ethics approval and consent to participate}

The present study was approved by the Ethics Committee of Shenzhen Maternity and Child Healthcare Hospital.

\section{Patient consent for publication}

Not applicable.

\section{Competing interests}

The authors declare that they have no competing interests.

\section{References}

1. Miller RD: Miller's anesthesia. Eighth edition. Philadelphia. Elsevier Saunders pp. 822-831, 2015.

2. Vanhorebeek I, Gunst J and Van den Berghe G: Critical care management of stress-induced hyperglycemia. Curr Diab Rep 18 17, 2018.

3. Van den Berghe G, Wilmer A, Hermans G, Meersseman W, Wouters PJ, Milants I, Van Wijngaerden E, Bobbaers H and Bouillon R: Intensive insulin therapy in the medical ICU. N Engl J Med 354: 449-461, 2006.

4. Yasuda Y, Fukushima Y, Kaneki M and Martyn JA: Anesthesia with propofol induces insulin resistance systemically in skeletal and cardiac muscles and liver of rats. Biochem Biophys Res Commun 431: 81-85, 2013.

5. Zhou L, Wang L, Yang B, Zeng J, Zhang Q, Lei H and Xu S: Protective effect of pretreatment with propofol against tumor necrosis factor- $\alpha$-induced hepatic insulin resistance. Exp Ther Med 10: 289-294, 2015.

6. Milne SE, Troy A, Irwin MG and Kenny GN: Relationship between bispectral index, auditory evoked potential index and effect-site EC50 for propofol at two clinical end-points. $\mathrm{Br}$ J Anaesth 90: 127-131, 2003.

7. Smith C, McEwan AI, Jhaveri R, Wilkinson M, Goodman D, Smith LR, Canada AT and Glass PS: The interaction of fentanyl on the $\mathrm{Cp} 50$ of propofol for loss of consciousness and skin incision. Anesthesiology 81: 820-828; Discussion 26A, 1994.

8. Hsing CH, Chen YH, Chen CL, Huang WC, Lin MC, Tseng PC, Wang CY, TsaiCC,ChoiPC andLinCF: Anesthetic propofolcauses glycogen synthase kinase-3 $\beta$-regulated lysosomal $/ \mathrm{mitochondrial}$ apoptosis in macrophages. Anesthesiology 116: 868-881, 2012.

9. Driessen GJ, IJspeert H, Wentink M, Yntema HG, van Hagen PM, van Strien A, Bucciol G, Cogulu O, Trip M, Nillesen W, et al: Increased PI3K/Akt activity and deregulated humoral immune response in human PTEN deficiency. J Allergy Clin Immunol 138: 1744-1747.e5, 2016.
10. Yue S, Li J, Lee SY, Lee HJ, Shao T, Song B, Cheng L, Masterson TA, Liu X, Ratliff TL and Cheng JX: Cholesteryl ester accumulation induced by PTEN loss and PI3K/AKT activation underlies human prostate cancer aggressiveness. Cell Metab 19: 393-406, 2014.

11. Bleau AM, Hambardzumyan D, Ozawa T, Fomchenko EI, Huse JT, Brennan CW and Holland EC: PTEN/PI3K/Akt pathway regulates the side population phenotype and ABCG2 activity in glioma tumor stem-like cells. Cell Stem Cell 4: 226-235, 2009.

12. Wishart MJ and Dixon JE: PTEN and myotubularin phosphatases: From 3-phosphoinositide dephosphorylation to disease. Trends Cell Biol 12: 579-585, 2002.

13. Elbashir SM, Harborth J, Lendeckel W, Yalcin A, Weber K and Tuschl T: Duplexes of 21-nucleotide RNAs mediate RNA interference in cultured mammalian cells. Nature 411: 494-498, 2001.

14. Shi XP, Zong AN, Tao J and Wang LZ: Study of instructive notions with respect to caring for laboratory animals. J China Med Univ 36: 493, 2007.

15. Seglen PO: Preparation of isolated rat liver cells. Methods Cell Biol 13: 29-83, 1976.

16. Casciano DA: Development and utilization of primary hepatocyte culture systems to evaluate metabolism, DNA binding, and DNA repair of xenobiotics. Drug Metab Rev 32: 1-13, 2000.

17. Livak KJ and Schmittgen TD: Analysis of relative gene expression data using real-time quantitative PCR and the 2(-Delta Delta C(T)) method. Methods 25: 402-408, 2001.

18. Lebovitz HE: Insulin resistance: Definition and consequences. Exp Clin Endocrinol Diabetes 109 (Suppl 2): S135-S148, 2001.

19. Reaven GM: Banting lecture 1988. Role of insulin resistance in human disease. Diabetes 37: 1595-1607, 1988.

20. Fahy BG, Sheehy AM and Coursin DB: Glucose control in the intensive care unit. Crit Care Med 37: 1769-1776, 2009.

21. van den Berghe G, Wouters P, Weekers F, Verwaest C, Bruyninckx F, Schetz M, Vlasselaers D, Ferdinande P, Lauwers P and Bouillon R: Intensive insulin therapy in critically ill patients. N Engl J Med 345: 1359-1367, 2001.

22. Li J, Yen C, Liaw D, Podsypanina K, Bose S, Wang SI, Puc J, Miliaresis C, Rodgers L, McCombie R, et al: PTEN, a putative protein tyrosine phosphatase gene mutated in human brain, breast, and prostate cancer. Science 275: 1943-1947, 1997.

23. Steck PA, Pershouse MA, Jasser SA, Yung WK, Lin H, Ligon AH, Langford LA, Baumgard ML, Hattier T, Davis T, et al: Identification of a candidate tumour suppressor gene, MMAC1, at chromosome 10q23.3 that is mutated in multiple advanced cancers. Nat Genet 15: 356-362, 1997.

24. Li DM and Sun H: TEP1, encoded by a candidate tumor suppressor locus, is a novel protein tyrosine phosphatase regulated by transforming growth factor beta. Cancer Res 57: 2124-2129, 1997.

25. Chu EC and Tarnawski AS: PTEN regulatory functions in tumor suppression and cell biology. Med Sci Monit 10: RA235-RA241, 2004.

26. Sha SH, Chen FQ and Schacht J: PTEN attenuates PIP3/Akt signaling in the cochlea of the aging CBA/J mouse. Hear Res 264: 86-92, 2010.

27. Leslie NR, Bennett D, Lindsay YE, Stewart H, Gray A and Downes CP: Redox regulation of PI 3-kinase signalling via inactivation of PTEN. EMBO J 22: 5501-5510, 2003. 